\title{
Excision of an intramuscular hemangioma within the anterior muscle compartment of the leg in a thirteen-year- old female: A case report
}

\author{
Jason Snyder ${ }^{1}$, Mark Mendeszoon $^{2 *}$ and Elizabeth Sanders ${ }^{3}$ \\ ${ }^{1}$ Attending Surgeon, Private Practice, $1501 \mathrm{~W}$ 6th St Suite 1A, Brooklyn NY, USA \\ ${ }^{2}$ Attending Surgeon and Fellowship Director, Precision Orthopaedic Specialties, Inc, Chardon, OH, USA \\ ${ }^{3}$ Foot and Ankle Surgical Fellow, Precision Orthopaedic Specialties, Inc, Chardon, OH, USA
}

\begin{abstract}
We report a 13-year-old female presenting with a painful soft tissue mass to the anterior aspect of the leg. Pain had gradually progressed to the point of preventing normal daily function. Conservative therapy of monitoring the lesion, compression wraps and saline deep tissue massage for 12-months duration failed. MRI examination revealed a soft tissue mass of unknown origin. At this time, surgical excision of the mass was performed. Pathology studies verified the mass was an intramuscular hemangioma involving the extensor digitorum longus, extensor hallucis longus, and tibialis anterior muscles. We document the surgical procedure and the post-operative care for this patient. To the author's knowledge, such an excision of an intramuscular hemangioma extending into three muscles of this location has not been presented in the literature.
\end{abstract}

\section{Introduction}

Hemangiomas are the most common benign soft tissue mass in children under age ten. The exact etiology is unknown. They are more prevalent in females than males and can be located anywhere throughout the body. Usually hemangiomas occur in the first few weeks of infancy and self-dissolve in the first decade. Intramuscular hemangiomas are very rare, accounting for less than $1 \%$ of all hemangiomas. They are difficult to diagnose with radiographic testing. Magnetic resonance imaging (MRI) may, as in this case, likely suspect a malignant process. Generally the patient will present with soft tissue swelling or mass with pain or discomfort in the area, typically for a duration of over one year. Conservative treatment may include immobilization, ice therapy, compression or deep tissue message. Surgically, complete excision of the hemangioma and hemostasis of associated blood vessels is recommended with complete pathology and histology work-up of the mass. Alternatively, the patient may elect to attempt sclerosing injections or endovascular embolization.

Microscopically, hemangiomas have a unique appearance. Intramuscular hemangiomas may be of 3 types: capillary, cavernous, or mixed. Intramuscular hemangiomas do not have nuclear hyperchromatism nor do they develop the freely anastomosing sinusoidal pattern as seen in certain angiosarcomas. Hemangiomas lack lipoblasts as seen in angiolipomas. Hemangiomendotheliomas display possible shedding and cellular atypia which is not seen in hemangiomas. Hemangiomas can be differentiated from angiolipomas by the absence of lipoblasts in hemangiomas.

\section{Case report}

A 13-year-old female presented to her podiatrist, the co-author, at his institution. She complained of a painful mass to the anterior aspect of her leg. She described the pain as aching and throbbing exacerbated by athletic activity, present for a duration of over one year. She had not sought previous treatment from a family practitioner. Previous treatments included ice, rest and elevation but did not alleviate symptoms. The patient noted that the mass had grown in size over the past several months which was concerning. Her past medical history, family history and social history were all unremarkable.

The physical exam was unremarkable except for a palpable mass on the lower leg. The mass did not transilluminate with light and the contralateral limb was $1 \mathrm{~cm}$ smaller in circumference at the point of the mass.

Plain film radiography displayed soft tissue swelling with no other obvious pathology. Magnetic resonance imaging (MRI) revealed a soft tissue mass coursing in the muscle bodies of tibialis anterior, extensor digitorum longus and extensor digitorum brevis.

At this point, treatment options were provided to the patient. Conservative therapeutic options included immobilization, icing and compression. Sclerosing injections were discussed but the patient declined due to fear of needles. Further options included leaving the lesion alone, or surgical excision. After exhausting all conservative measures, the patient decided to proceed with surgical excision.

Upon extraction of the lesion, specimens were sent to pathology and microbiology for study. A Jackson-Pratt 5.0 drain was placed to aid in evacuation of any fluid. A plastics closure was utilized due to

Correspondence to: Mark Mendeszoon, Attending Surgeon and Fellowship Director, Precision Orthopaedic Specialties, Inc, 150 7th Ave \#200, Chardon, OH 44024, USA, E-mail: dr1zoom@roadrunner.com

Received: December 04, 2017; Accepted: December 15, 2017; Published: December 19, 2017 
location and age of the patient. The patient was placed in a modified Jones dressing with a posterior splint.

Post-operative course consisted of a non-weightbearing Jones compression dressing for 1 week, non-weightbearing below knee cast for 2 weeks, and finally partial weightbearing with a high-tide walking boot for three weeks. She was placed on post-operative pain medications and antibiotics. After 3 weeks, she began scar therapy including rigorous cross fiber message with lotion. Physical therapy began in earnest which included range of motion and muscle strengthening exercises.
At 8 weeks, she returned to athletic activity without pain or limitation. The patient is currently 8 years removed from surgery and completely symptom free [1-9] (Figures 1-7).

\section{Discussion}

Histology and pathology studies demonstrated the lesion to be a benign intramuscular hemangioma. As this lesion has a substantial recurrence rate from $18-61 \%$, it is imperative that deliberate, delicate and wide excision margins be completed to improve long-term outcome for the patient.

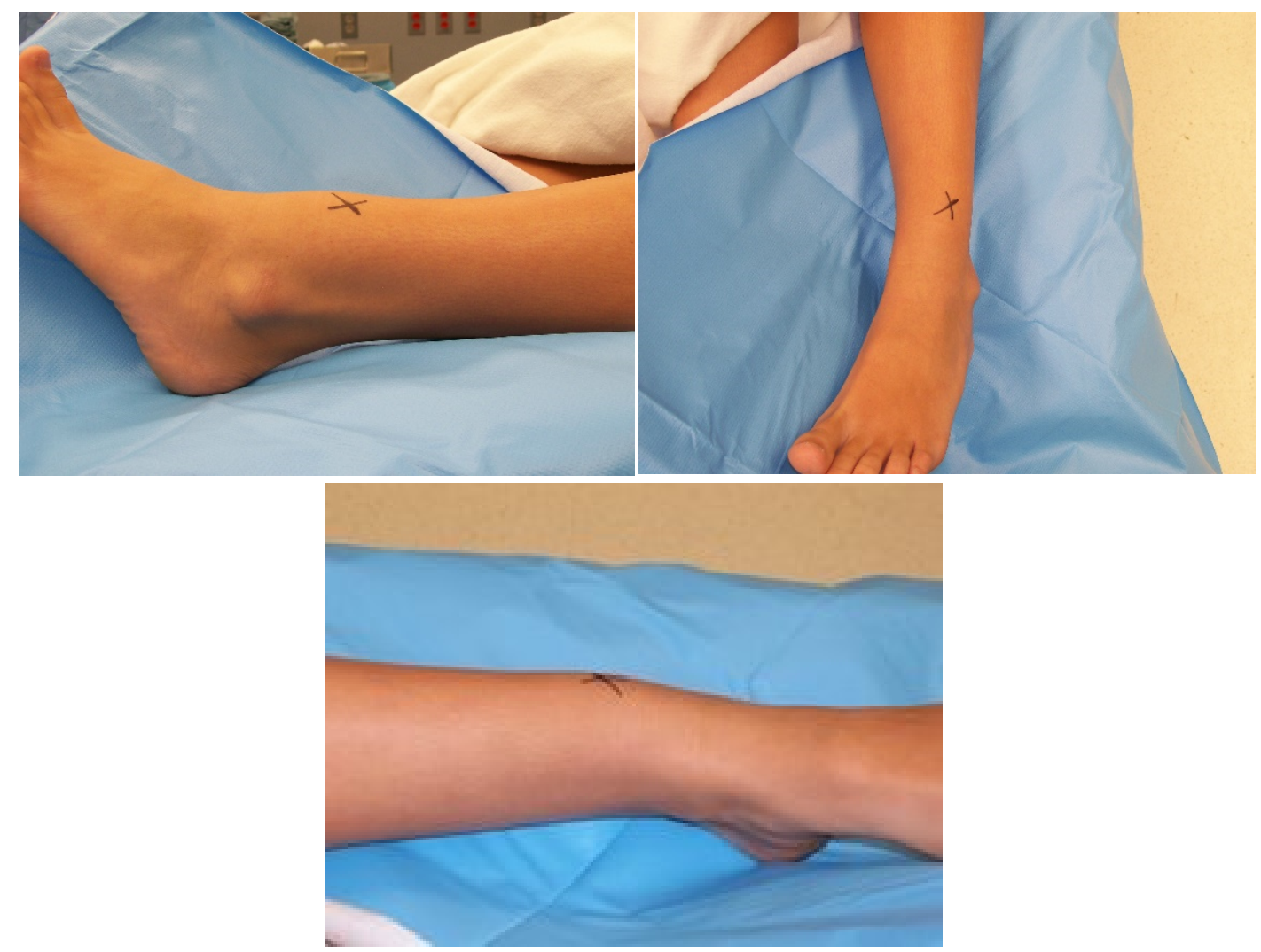

Figure 1. Three gross views of the mass in the anterior aspect of the leg.

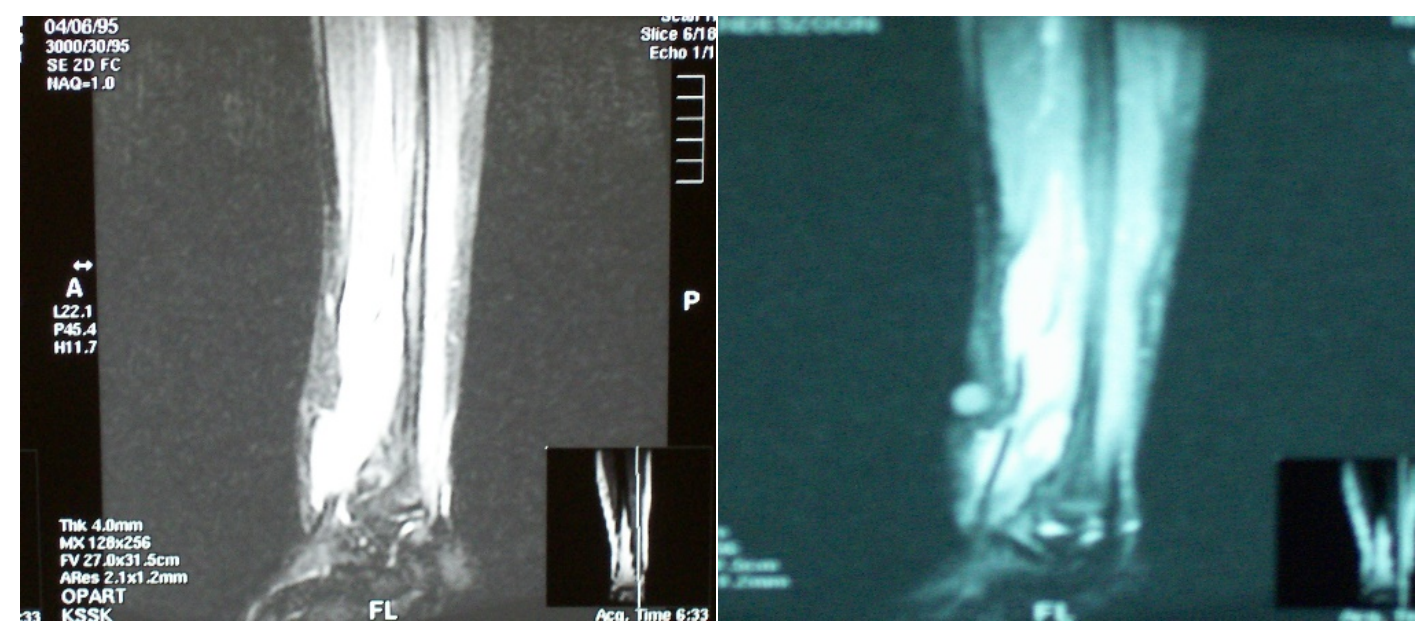

Figure 2. Magnetic Resonance Imaging displaying the poorly defined lesion with high signal intensity. Note the extent and size of the lesion. 


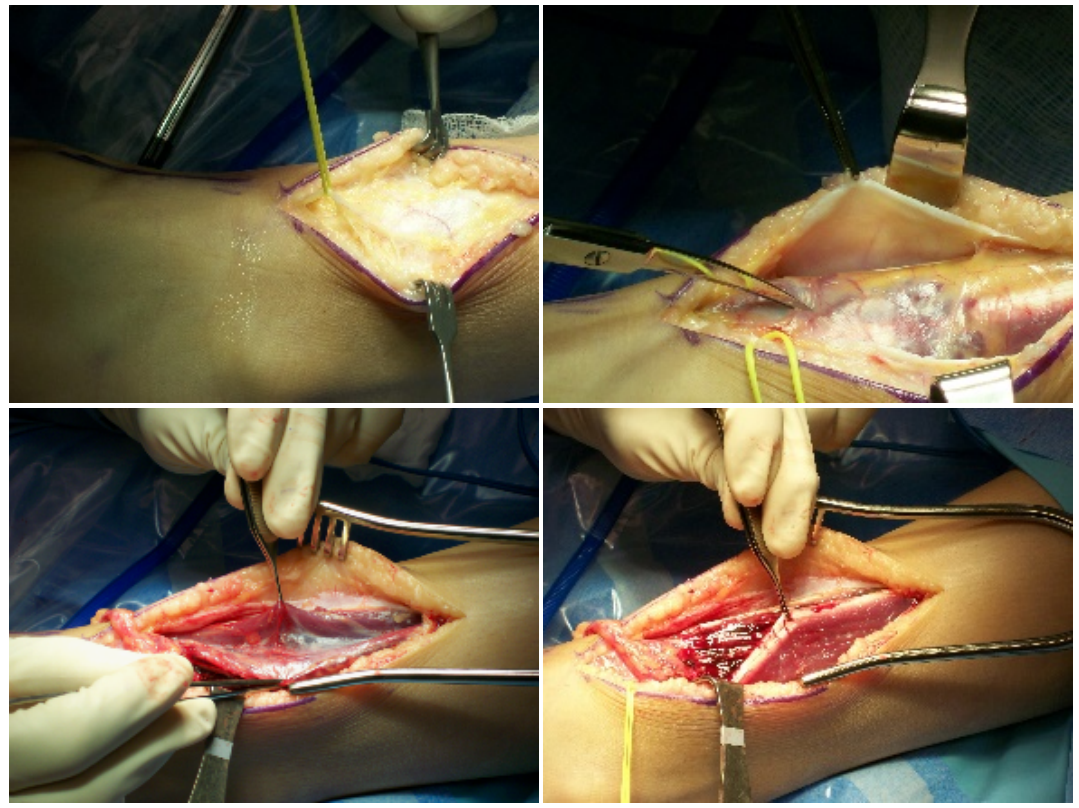

Figure 3. Layered dissection through to the level of the hemangioma location.

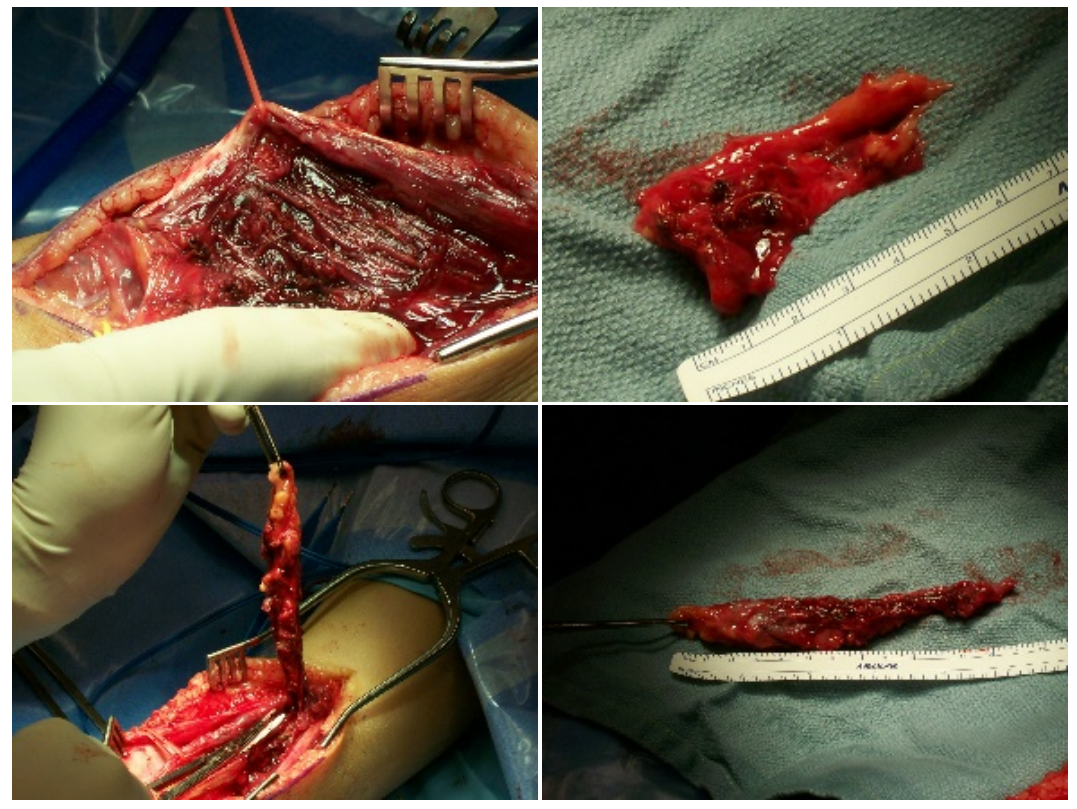

Figure 4. Excision of the hemangioma.

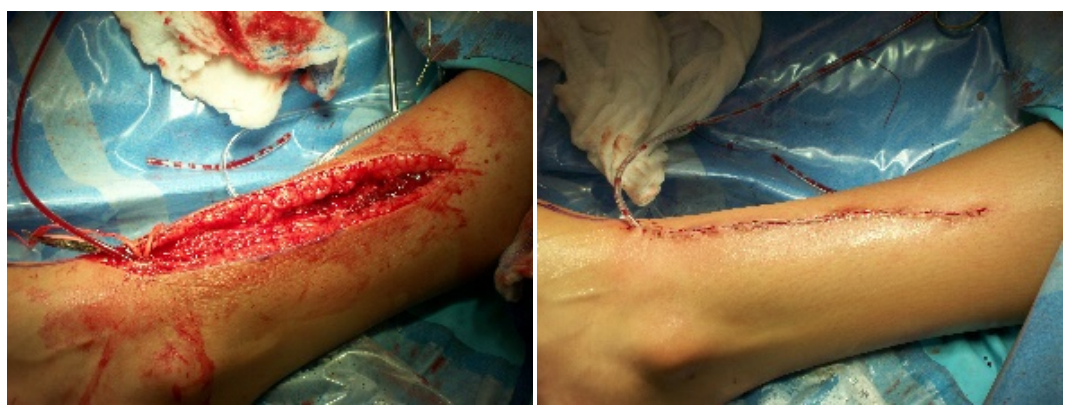

Figure 5. Closure of the incision with insertion of a 5.0 Jackson-Pratt drain. 


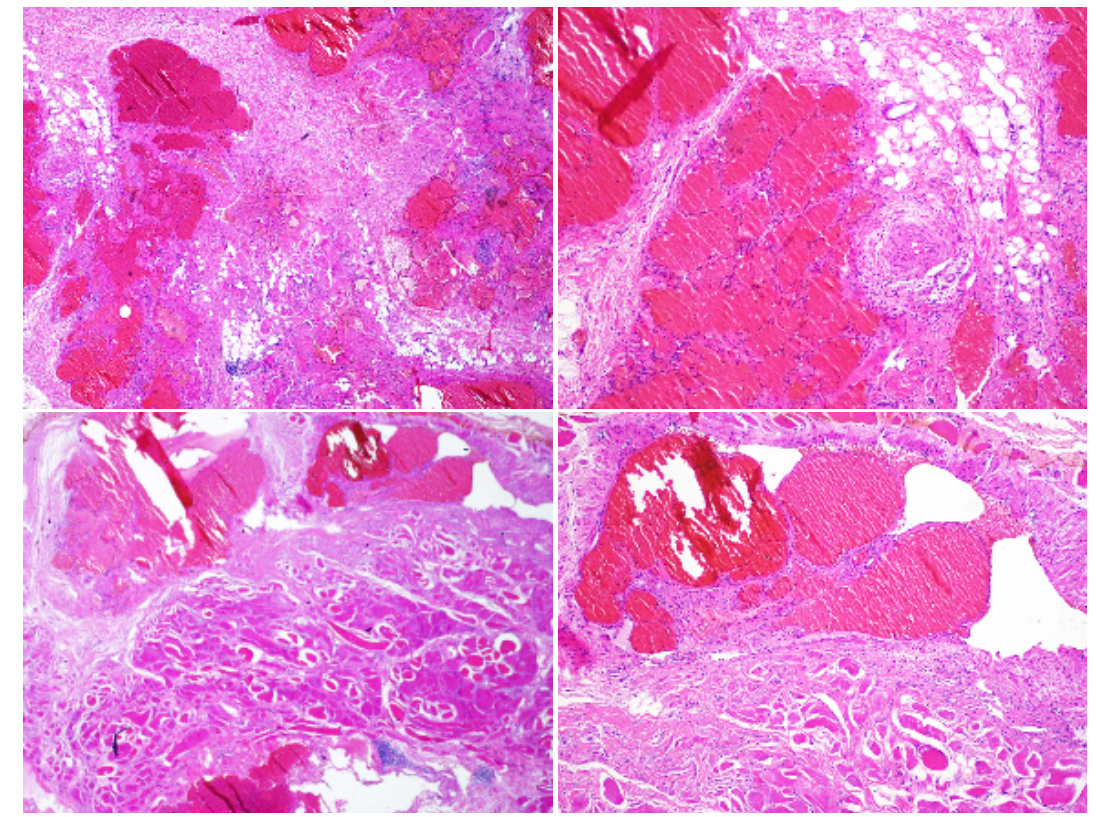

Figure 6. Histology slides revealing a benign intramuscular hemangioma of the anterior muscles compartment of the leg.

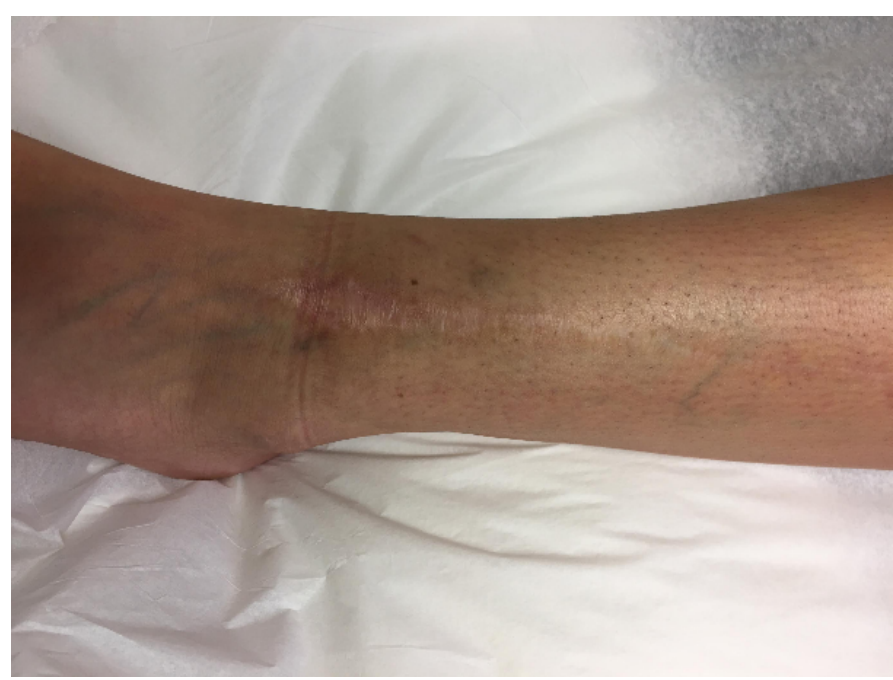

Figure 7. Clinical picture eight years post-operatively.

\section{References}

1. Cohen AJ, Youkey JR, Clagett GP, Huggins M, Nadalo L, et al. (1983) Intramuscular hemangioma. JAMA 249: 2680-2682. [Crossref]

2. Kistler B, Damron T (2016) Orthopedic surgery for hemangioma.

3. Lakshmi K, Sankarapandiyan S, Mohanarangam V (2014) Intramuscular haemangioma with diagnostic challenge: a case for strange pain in the masseter muscle. Case Rep Dent [Crossref].

4. Management of infantile hemangioma (2016) Vanderbilt University Evidence-based Practice Center for the Agency for Healthcare Research and Quality, January 2016. www.effectivehealthcare.ahrq.gov/infantile-hemangioma.

5. Melman L, Johnson FE (2008) Intramuscular cavernous hemangioma. Am J Surg 195 816-817. [Crossref]

6. Oumakhir S, Ghfir M, El Ktaibi A, Albouzidi A, Sedrati O (2010) Synovial hemangioma of the ankle. Foot (Edinb) 20: 42-43. [Crossref]

7. Shankar V (2016) Soft tissue benign vascular tumors. Intramuscular hemangioma. Pathology Outlines.com, Inc.

8. Vilanova JC, Barceló J, Smirniotopoulos JG, Pérez-Andrés R, Villalón M, et al. (2004) Hemangioma from head to toe: MR imaging with pathologic correlation. Radiographics 24: 367-385. [Crossref]

9. Wierzbicki JM, Henderson JH, Scarborough MT, Bush CH, Reith JD, et al. (2013) Intramuscular hemangiomas. Sports Health 5: 448-454. [Crossref]

Copyright: @2017 Snyder J. This is an open-access article distributed under the terms of the Creative Commons Attribution License, which permits unrestricted use, distribution, and reproduction in any medium, provided the original author and source are credited. 